\title{
The genesis of cystic fibrosis lung disease
}

\author{
Jeffrey J. Wine \\ Cystic Fibrosis Research Laboratory, Stanford University, Room 450, Bldg. 420, Main Quad, \\ Stanford, California 94305-2130, USA. Phone: (650) 725-2462; Fax: (650) 725-5699; \\ E-mail:wine@stanford.edu
}

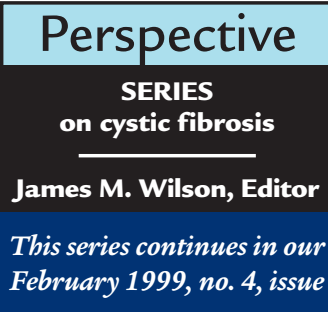

Cystic fibrosis (CF), as Bals et al. discuss in the other Perspective in this issue (1), is one of the most thoroughly understood genetic diseases. Thus it is frustrating that CF lung disease has been refractory to the onslaught of molecular/cellular research that has clarified so many other aspects of CF. But after many false starts, recent experiments are finally beginning to crack the shell of ignorance that surrounds CF lung disease. A series of innovative experiments, mainly from two laboratories, has revealed fascinating new aspects of normal and CF lung biology. Yet despite the care and resourcefulness of each group, their findings generate sharply contrasting views of CF lung disease that lead to opposite treatment strategies! Fortunately, this controversy is generating more light than heat and is illuminating the field to the ultimate benefit of CF clinical care.

CF lung disease. Healthy airways are sterile below the first bronchial division. Sterility is maintained, despite constant challenge from viruses and bacteria in the air that we breathe, by an elaborate hierarchy of defenses. Lung defenses are understood only in outline. The airway surfaces are covered with a thin film $(\sim 30 \mu \mathrm{m})$ of airway surface liquid (ASL) consisting of a periciliary sol and a mucus gel that are propelled toward the mouth by coordinated ciliary beating. Thus, mucociliary clearance, aided by cough, cleans the airways mechanically. The ASL is not simply saltwater, but is instead a rich broth of proteases/antiproteases, oxidants/antioxidants, antibiotics, and antibodies that work together to inactivate or destroy pathogens without undue collateral damage to the lungs. These mucosal mechanisms are backstopped by cellular immune mechanisms involving dendritic cells (2), neutrophils, and macrophages that are recruited and coordinated by signaling molecules in the ASL.

Although the complex pulmonary defense system is understood in outline, details are lacking because of technical impediments that, together with the lack of appropriate animal models, help to explain why we are still struggling to understand $\mathrm{CF}$ lung disease. The pattern of CF lung disease is unlike that of any other lung disease. In $\mathrm{CF}$, infections of the smaller airways are nearly universal, difficult and eventually impossible to eradicate, and almost invariably lethal. The types of organisms that most bedevil CF patients, such as Pseudomonas aeruginosa or Burkbolderia cepacia, are innocuous in normal individuals - to such an extent that they are being sprayed into the environment for various biotechnical applications. Disease is most severe in the upper lobes and involves huge infiltrations of neutrophils.

Functions of CFTR. As explained in more detail by Bals et al., CFTR, the product of the gene that is defective in CF, is a member of the ATP Binding Cassette (ABC) family. $\mathrm{ABC}$ family members have diverse functions, including ATP-dependent transmembrane pumping of larger molecules, regulation of other membrane transporters, and ion conductance. CFTR displays at least two of these functions. CFTR is an anion channel that uses the energy of ATP hydrolysis to transit through conducting and non-conducting conformations. Ion channels are equally adept at allowing ions to flow either into or out of cells, enabling the CFTR anion channel to play crucial roles in both absorption and secretion. Thus the loss of CFTRmediated anion conductance explains a wide range of $\mathrm{CF}$ symptoms, such as elevated sweat chloride (a defect in salt absorption by the sweat ducts) and meconium ileus (a defect in fluid secretion by intestinal crypt cells).

CFTR can also regulate other membrane proteins (3). The most thoroughly documented regulatory role for CFTR is its negative regulation of the amiloride-sensitive epithelial $\mathrm{Na}^{+}$channel (ENaC). CFTR decreases $\mathrm{ENaC}$ 's open probability $(\mathrm{Po})$ and reverses its usual increases to elevations of [cAMP $]_{i}$ (4). When CFTR function is lost, the $\mathrm{Na}^{+}$conductance is markedly increased in CF human airways (5) and CF mouse nasal epithelia (6), but apparently not in human sweat ducts (7). CFTR's channel and regulatory (8) functions require that it be phosphorylated by various kinases, especially cAMP-dependent protein kinase (PKA).

To summarize, CFTR is an anion channel and a channel regulator that plays multiple roles in epithelial transport. Although other functions have been proposed for CFTR, most present hypotheses of CF disease emphasize CFTR's roles in ion transport (Fig. 1).

From CFTR to lung disease. The vexing problem of explaining CF lung disease using known properties of CFTR has recently been energized by two innovative but sharply different hypotheses. Interestingly, both hypotheses consider CFTR's role in salt absorption to be primary. The high salt hypothesis $(9,10)$ emphasizes CFTR's function as an anion channel. According to this hypothesis, missing or defective CFTR causes reduced transepithelial $\mathrm{Cl}^{-}$con- 


\section{Figure 1}

CFTR's multiple roles in fluid and electrolyte transport. (a) Salt absorption. In the sweat duct, high apical conductance for $\mathrm{Na}^{+}[1]$ and $\mathrm{Cl}^{-}[2]$ and relatively low water conductance allows salt to be reabsorbed in excess of water [hypertonic absorption) leaving a hypotonic luminal fluid. In the sweat duct CFTR is the only available anion conductance pathway, and when it is lost in CF the lumen quickly becomes highly electronegative and transport virtually ceases, resulting in high (similar to plasma) luminal salt $(\boldsymbol{b})$. Fluid absorption. In epithelia with high water per-

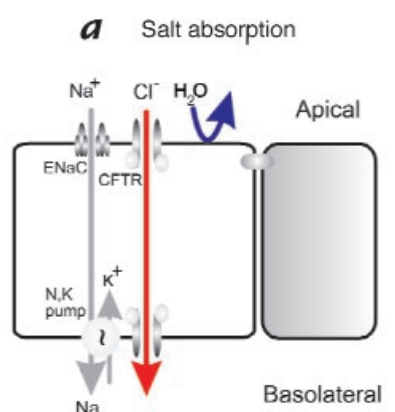

[1] [2]

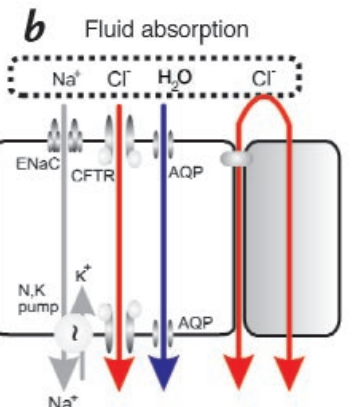

[1] [2] [3]

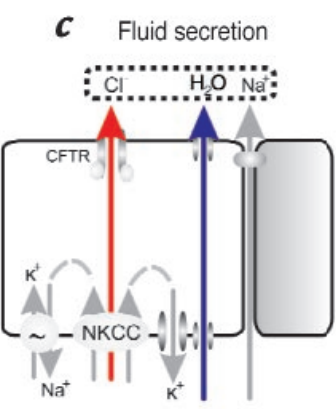

[5] [6][7]

meability [3] relative to electrolyte permeability water will absorbed osmotically with salt to decrease the volume of luminal fluid. If no other osmolytes or forces are present, the salt concentration will remain unchanged. If water-retaining forces are present, permeant electrolytes can be reduced preferentially. The consequences of eliminating CFTR depend on the magnitude of such forces, the relative magnitude of alternate pathways for transepithelial anion flow [4], and how CFTR affects other ion channels. The high salt and low volume hypotheses differ on each of these points. (c) Anion-mediated fluid secretion. Secreting epithelia lack a significant apical $\mathrm{Na}^{+}$conductance. Basolateral transporters such as $\mathrm{NKCC}$ move $\mathrm{Cl}^{-}$uphill into the cell; it then flows passively into the lumen via CFTR [5], $\mathrm{K}^{+}$exits basolaterally, $\mathrm{Na}^{+}$flows paracellularly [7] and water follows transcellularly [6]. Elimination of CFTR eliminates secretion.

ductance, and by analogy with the sweat duct $(11,12)$ this allows salt levels in the ASL to remain at levels similar to those in plasma. The high salt in the ASL interferes with natural antibiotics such as defensins (13) and lysozyme.

In marked contrast, the low volume hypothesis (14) is based on CFTR's function as a regulator of other channels - in this case ENaC. According to this hypothesis, both normal and CF ASL have plasma-like levels of salt. CFTR mutations eliminate CFTR's inhibition of ENaC, and because there are significant shunt pathways for $\mathrm{Cl}^{-}$ in the airways, increased $\mathrm{Na}^{+}$transport drives increased absorption of $\mathrm{Cl}^{-}$and water. Thus CF airways display accelerated isotonic fluid absorption that depletes ASL volume and dehydrates mucus, leading to obstruction and infection (15).

How have these two disparate views arisen?

The high salt bypothesis. In a clever series of experiments, Smith et al. (9) obtained airway epithelial cells from normal and CF individuals and grew them on filters until they formed monolayers of cells joined by tight junctions. Bacteria were then placed into the fluid covering the apical surfaces of the cultured cells. The dramatic finding was that bacteria flourished in their cultures of CF airway cells, but were killed by their cultures of normal cells! Bacteria placed in the media bathing the basolateral surface of the cells flourished for both kinds of cells, suggesting that normal cultured airway cells produce apical factors that kill bacteria. A further surprise occurred when the salt content of the apical fluid was manipulated. Merely adding pure water to the fluid from CF cells rendered it bactericidal; merely adding salt $(\mathrm{NaCl})$ to fluid from normal cells allowed bacteria to flourish! Smith et al. concluded that both normal and CF airway epithelial cells liberate antibiotics into the ASL, but in the ASL of CF cultures the antibiotics are rendered ineffective by a higher salt concentration (9).

These findings galvanized the CF community. They catapulted the field of mucosal defenses to the forefront of CF research and provided an exciting new look at the question of CF lung disease. They also posed a direct challenge to the competing hypothesis of hyperabsorption by CF airways, and helped provoke intense examination of how airways produce and condition ASL.
Is salt higher in the apical fluid of CF epithelial cultures relative to normal cultures, and if so why? Zabner et al. (10) developed a radioisotopic method to measure salt levels in the tiny volumes of surface fluid in their air interface cultures. Apical fluid covering normal cells had levels of $\mathrm{Na}^{+}$and $\mathrm{Cl}^{-}$that were reduced by $\sim 2 / 3$ relative to the basolateral fluid, indicating a significant absorption of salt in excess of water; fluid volume was unchanged. In contrast, apical fluid covering CF cells was approximately twice as salty as normal.

The authors suggested that their results might be analogous to failure of salt reabsorption in the CF sweat duct ( 9 , 10 ), where $80 \%-90 \%$ of the salt is reabsorbed. The ability of the ducts to absorb salt in excess of water is a remarkable feature shared by few epithelia, because it requires that charged ions permeate the epithelium more easily than water. It is made possible in the sweat duct by its extremely high electrolyte permeability - at $125 \mathrm{mS} / \mathrm{cm}^{2}$ it is among the highest of any known epithelium (11) - and probably by low membrane water permeability, although that has not been measured. Normal ducts contain open CFTR and $\mathrm{ENaC}$ channels to facilitate the transcellular movement of $\mathrm{NaCl}$. In CF sweat ducts, salt absorption fails because of a loss of $\mathrm{Cl}^{-}$conductance $(11,12)$, secondary to the loss of CFTR channels (e.g. 15). The ducts contain no other pathway for $\mathrm{Cl}^{-}$, so that the transfer of a tiny proportion of $\mathrm{Na}^{+}$ ions out of the CF lumen produces a marked lumen negativity that prevents further $\mathrm{Na}^{+}$movement.

Smith et al. propose that normal airways, like sweat ducts, reabsorb salt in excess of water from the airway surface liquid, thus producing a low salt content of ASL that is required for normal bacterial killing by natural antibiotics, (Fig. 2a). In CF this process is defective $(9,10)$ (Fig. 2b).

The low volume hypothesis. Based on elegant experiments, Boucher and colleagues come to a markedly different conclusion (14). They also grew airway epithelial cell cultures on filters, using procedures that produced well-differentiated cultures. But they found no difference in either the salt content or the osmolarity of surface liquid from normal and CF airway cultures, both of which were isosmotic relative to basolateral fluid (Fig. 2c). Instead, the liquid layer in their CF epithelial cultures became reduced in volume, and mucus 
movement was impeded. These findings fit with their extensive evidence for $\mathrm{Na}^{+}$hyperabsorption in CF epithelia, and provide direct evidence for one of the earliest hypotheses to explain CF lung disease, the "thick mucus" hypothesis.

Animal airways have a moderate osmotic water permeability and express aquaporin channels in their basolateral membranes (16). Less is known about human airways. Matsui et al. assayed the water permeability of their human airway cultures by placing hypotonic phosphate-buffered saline (PBS) onto the apical surface. They then measured osmolality over time, as well as changes in the volume of liquid at the surface and subsurface and the height of the epithelial cell. The hypotonic fluid rapidly ( $\sim 10 \mathrm{~min})$ equilibrated to isotonicity and its volume decreased with a corresponding increase in the volume of the cellular and subcellular compartments. MDCK cells, which have lower water permeability (17), showed no evidence for volume absorption. Additional experiments with cells on filters and cells grown in hollow "biofibers" indicate that airway cells grown in both ways absorb isotonic fluid, and that this absorption is $\mathrm{Na}^{+}$dependent because it stops when $\mathrm{Na}^{+}$is replaced with $N$-methyl-D-glucamine.

What happens in CF airways? The hypothesis is that lack of CFTR inhibition of ENaC causes increased $\mathrm{Na}^{+}$absorption, with $\mathrm{Cl}^{-}$following via shunt pathways and water flowing transcellularly, leading to decreased volume (Fig. 2d). In striking experiments, Matsui et al. demonstrate, for the first time, the consequences of hyperabsorption in CF airway cultures. Starting with a $30 \mu \mathrm{m}$ deep layer of ASL, they observed a reduction to $\sim 10 \mu \mathrm{m}$ over a 24 -hour period, with no increase in $\mathrm{Cl}^{-}$concentration. Their cultures secrete mucus, and after addition of fluid approximately $30 \%$ of their cultures show regions of rotational mucus transport. Mucus transport continued in normal cultures, but stopped after 24 hours in CF cultures. Inspection of the cultures with confocal and electron microscopy indicated that depletion of the sol layer in CF cultures allowed the mucus layer to collapse the cilia and stop transport

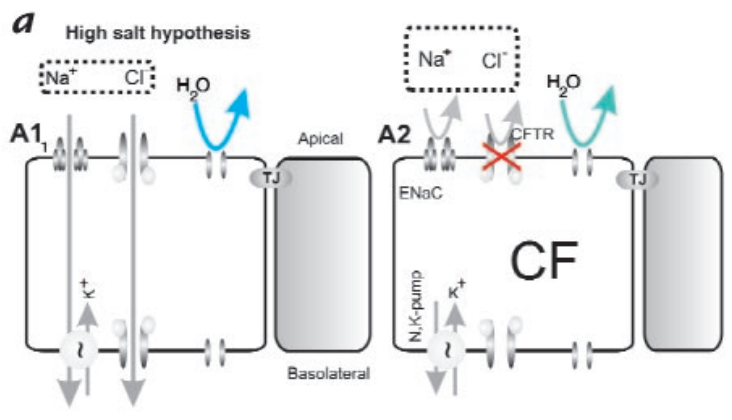

b Low volume hypothesis

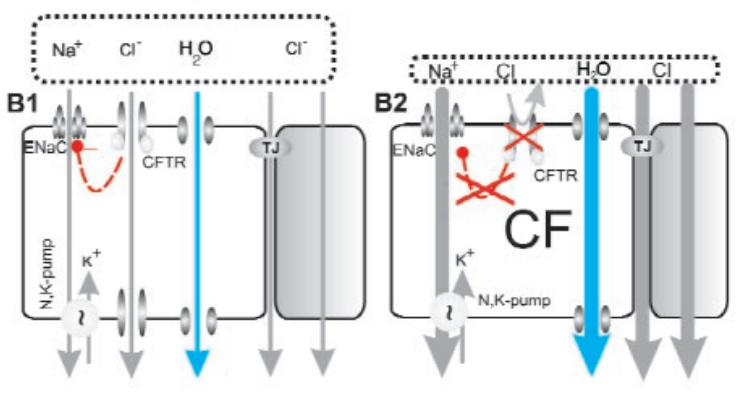

(14). If this happens in CF airways it should compromise mucociliary clearance and promote infection (14).

What is going on? According to Lyman Beecher, the respected minister, abolitionist, and father of Harriet Beecher Stowe, "No great advance has been made in science, politics or religion without controversy." If advances are proportional to the degree of controversy, then $C F$ research can anticipate a very great advance indeed, because the opposing views of airway fluid processing just reviewed could hardly differ more. How can such disparate results have arisen?

Some differences could arise from methodology, e.g., smaller ASL volumes are required for low salt (10). But each group used several different methods to gather evidence, making it less likely that systematic errors occurred. Instead, it seems more plausible that each group is accurately measuring the properties of their cell cultures, but that those properties differ. The cells giving high salt results were mixtures of nasal, tracheal and bronchial cells used $8-45$ days after seeding, with resistances [> $500 \mathrm{~m} \Omega \times \mathrm{cm}^{2}$ (9), or $\geq] 800 \Omega \times \mathrm{cm}^{2}$ (10). The cells giving low volume results were bronchial cells used $\sim 28$ days after seeding, with resistances $>300 \Omega \times \mathrm{cm}^{2}$ (14). Thus, although each group has produced high quality epithelial cell cultures by using variations of improved methods (e.g. 17), the basic descriptive data are insufficient to allow the conclusion that the cultures are comparable.

Of all the variables in these experiments, the cultured cells are by far the most complex, with many possible features that might differ. Cultured epithelial cells express different degrees of water permeability $(14,17)$, CFTR expression (19) and shunt resistance, and the proportions of different cell types in these highly non-homogeneous cultures can also differ. Such features profoundly affect epithelial transport properties and the consequences of CFTR malfunction. For example, inspection of Fig. $2 b$ makes it clear that the low volume hypothesis requires a non-CFTR pathway for transcellular anion flow. The shunt pathway shown is paracellular, but it could also include pathways through a different cell type, as occurs in frog skin. If this shunt pathway is large relative to the CFTR-mediated transcellular pathway and equal in CF and normal tissues, then CF tissues will absorb more because of higher ENaC activity. But if the shunt pathway is small relative to the CFTR-mediated tran-

\footnotetext{
Figure 2

Two hypotheses of how airway surface liquid (ASL) differs in healthy and CF lungs. (a) The high salt hypothesis $(9,10)$ postulates that normal ASL has low levels of salt as a result of salt absorption in excess of water (A1, left). Even though the epithelium is water permeable, salt is retained in thin surface films by some combination of surface tension (28) and impermeant osmolytes (10). In CF (A2), salt is poorly absorbed resulting in excessively salty ASL that disrupts natural mucosal antibiotics. Key features of the high salt model are: the lack of an appreciable shunt $\mathrm{Cl}^{-}$conductance, central importance of CFTR's channel role, no specific role for inhibition of ENaC by CFTR, and a switch from isotonic volume absorption to hypertonic salt absorption as the surface layer thins and traps residual water. (b) The low volume hypothesis (14) postulates that normal ASL (B1) has salt levels approximately equal to plasma. In $\mathrm{CF}(B 2)$, the removal of CFTR's inhibition of $\mathrm{ENaC}$ results in abnormally elevated isotonic fluid absorption which depletes the ASL and leads to reduced mucociliary clearance. Key features of the low volume model are the parallel pathway for $\mathrm{Cl}^{-}$via shunt pathway(s) and inhibition of ENaC via CFTR.
} 
scellular pathway in normal tissues, they are predicted to absorb more than CF tissues (19). To continue this catalog of possible differences, in epithelia with high water permeability, maintenance of an osmotic gradient requires some additional force. Zabner et al. suggest that surface tension by some combination of cilia, mucus, or impermeant osmolytes might provide the necessary balancing force (10), but Matsui et al. provided data against a role for surface tension and did not find any evidence for osmolytes other than $\mathrm{NaCl}$ (14). Thus, it will be important to determine if the cultures differ with regard to water permeability, non-CFTR anion shunts, mucus production, osmolyte secretion, and ciliary beating.

What about the composition of ASL in actual human airways? Of course, what we really want to know about is actual airways. However, it isn't yet feasible to do the necessary experiments in vivo, which is why it is necessary to rely so heavily on experiments with cultured cells. There is no consensus on the ionic composition of normal and CF ASL in spite of $\sim 6$ studies cited in Zabner et al. (10) and Matsui et al. (14), because the volume of fluid to be sampled is tiny and is rapidly altered when disturbed. Only one study so far has used microanalytical (and hence minimally disruptive) methods, and it found that normal ASL had markedly lower $\mathrm{NaCl}$ and markedly higher $\mathrm{K}^{+}$concentrations than plasma, with an elevation to plasma-like concentrations in CF patients (20). On the other hand, patients with pseudohypoaldosteronism have disabled $\mathrm{ENaC}$ channels, and it was predicted that such patients would have high salt in their ASL and as a consequence show a CF-like disease (21). A preliminary report cited in (14) shows that these patients produce an abundant, isotonic ASL, and they do not appear to have a CF-like infectious airways disease.

What about secretion? It is remarkable that the two hypotheses of CF airway disease that dominate the present research field are focused on defects in absorption. Most symptoms of CF such as meconium ileus, loss of pancreatic function, degeneration of the vas deferens, thickened cervical mucus, and failure of adrenergically mediated sweating are attributed to CFTR's role in $\mathrm{Cl}^{-}$-driven fluid secretion (Fig 1c). In human lungs the highest levels of CFTR occur in serous cells of submucosal glands (22) and in scattered, non-ciliated surface cells in more distal airways (23) that may also be serous-like. Gland serous cells are abundant sources of bactericidal and antifungal compounds that probably are important for mucosal defenses. Animal (24) and cell culture models (25-27) of these poorly accessible cells indicate that CFTR is critical for glandular fluid secretion driven by $\mathrm{Cl}^{-}$and $\mathrm{HCO}_{3}^{-}$, and predict that the loss of gland function will exacerbate whatever absorptive defect is eventually found.

The controversy about ASL composition has benefited the CF community by focusing research onto the most malign consequence of CF disease. It has reinforced the need for scrupulous reporting of cell culture properties, for continued development of better model systems, and for novel approaches to in vivo measurements. It has emphasized that molecular/cellular methods are not sufficient for understanding many human diseases - we neglect organ system physiology at our peril. Finally, individuals directly affected by CF can take comfort in the knowledge that the insights produced by these studies are spawning a host of innovative treatments for CF lung disease, including increased use of inhaled antibiotics and renewed approaches to enhance CF lung clearance.

1. Bals, R., Weiner, D.J., and Wilson, J.M. 1999. The innate immune system in cystic fibrosis lung disease. J. Clin. Invest. 103:303-307.

2. McWilliam, A.S., et al. 1996. Dendritic cells are recruited into the airway epithelium during the inflammatory response to a broad spectrum of stimuli.J. Exp. Med. 184:2429-2432.

3. Egan, M., et al. 1992. Defective regulation of outwardly rectifying Clchannels by protein kinase A corrected by insertion of CFTR. Nature. 358:581-584.

4. Stutts, M.J., Rossier, B.C., and Boucher, R.C. 1997. Cystic fibrosis transmembrane conductance regulator inverts protein kinase A-mediated regulation of epithelial sodium channel single channel kinetics. J. Biol. Chem. 272:14037-14040

5. Knowles, M.R., et al. 1983. Abnormal ion permeation through cystic fibrosis respiratory epithelium. Science. 221:1067-1070.

6. Grubb, B.R., Vick, R.N., and Boucher, R.C. 1994. Hyperabsorption of $\mathrm{Na}^{+}$and raised $\mathrm{Ca} 2+-$-mediated $\mathrm{Cl}-$ secretion in nasal epithelia of CF mice. Am. J. Physiol. 266:1478-1483.

7. Reddy, M.M., and Quinton, P.M. 1997. Coordinated regulation of amiloride sensitive $\mathrm{Na}^{+}$and $\mathrm{CFTR} \mathrm{Cl-conductances} \mathrm{by} \mathrm{PKA} \mathrm{phospho-}$ rylation in the native sweat duct. Ped. Pulm. 14 (Suppl.):230-231.

8. Stutts, M.J., et al. 1995. CFTR as a cAMP-dependent regulator of sodium channels. Science. 269:847-850.

9. Smith, J.J., Travis, S.M., Greenberg, E.P., and Welsh, M.J. 1996. Cystic fibrosis airway epithelia fail to kill bacteria because of abnormal airway surface fluid. Cell. 85:229-236.

10. Zabner, J., Smith, J.J., Karp, P.H., Widdicombe, J.H., and Welsh, M.J. 1998. Loss of CFTR chloride channels alters salt absorption by cystic fibrosis airway epithelia in vitro. Mol. Cell. 2:397-403.

11. Quinton, P.M. 1986. Missing $\mathrm{Cl}$ conductance in cystic fibrosis. Am.J. Physiol. 251:C649-C652.

12. Quinton, P.M. 1983. Chloride impermeability in cystic fibrosis. Nature. 301:421-422.

13. Goldman, M.J., Anderson, G.M., Stolzenberg, E.D., Kari, U.P., Zasloff, M., and Wilson, J.M. 1997. Human beta-defensin-1 is a salt-sensitive antibiotic in lung that is inactivated in cystic fibrosis. Cell. 88:553-560.

14. Matsui, H., et al. 1998. Evidence for periciliary liquid layer depletion, not abnormal Ion composition, in the pathogenesis of cystic fibrosis airways disease. Cell. 95:1005-1015.

15. Kartner, N., Augustinas, O., Jensen, T.J., Naismith, A.L., and Riordan, J.R. 1992. Mislocalization of delta F508 CFTR in cystic fibrosis sweat gland. Nat. Genet. 1:321-327.

16. Verkman, A.S. 1998. Role of aquaporin water channels in kidney and lung. Am. J. Med. Sci. 316:310-320.

17. Farinas, J., Kneen, M., Moore, M., and Verkman, A.S. 1997. Plasma membrane water permeability of cultured cells and epithelia measured by light microscopy with spatial filtering. J. Gen. Physiol. 110:283-296.

18. Kondo, M., Finkbeiner, W.E., and Widdicombe, J.H. 1991. Simple technique for culture of highly differentiated cells from dog tracheal epithelium. Am. J. Physiol. 261:L106-L117.

19. Uyekubo, S.N., et al. 1998. cAMP-dependent absorption of chloride across airway epithelium. Am. J. Physiol. 275:L1219-L1227.

20. Joris, L., Dab, I., and Quinton, P.M. 1993. Elemental composition of human airway surface fluid in healthy and diseased airways. Am. Rev. Respir. Dis. 148:1633-1637.

21. Quinton, P.M. 1994. Viscosity versus composition in airway pathology. Am. J. Respir. Crit. Care Med. 149:6-7.

22. Engelhardt, J.F., et al. 1992. Submucosal glands are the predominant site of CFTR expression in the human bronchus. Nat. Genet. 2:240-248.

23. Engelhardt, J.F., Zepeda, M., Cohn, J.A., Yankaskas, J.R., and Wilson, J.M. 1994. Expression of the cystic fibrosis gene in adult human lung. J. Clin. Invest. 93:737-749.

24. Trout, L., King, M., Feng, W., Inglis, S.K., and Ballard, S.T. 1998. Inhibition of airway liquid secretion and its effect on the physical properties of airway mucus. Am. J. Physiol. 274:L258-L263.

25. Shen, B.Q., Finkbeiner, W.E., Wine, J.J., Mrsny, R.J., and Widdicombe, J.H. 1994. Calu-3: a human airway epithelial cell line that shows cAMPdependent Cl-secretion. Am. J. Physiol. 266:L493-L501.

26. Lee, M.C., Penland, C.M., Widdicombe, J.H., and Wine, J.J. 1998. Evidence that Calu-3 human airway cells secrete bicarbonate. Am. J. Physiol. 274:L450-L453.

27. Moon, S., Singh, M., Krouse, M.E., and Wine, J.J. 1997. Calcium-stimulated $\mathrm{Cl}-$ secretion in Calu-3 human airway cells requires CFTR. Am. J. Physiol. 273:L1208-L1219.

28. Widdicombe, J.H., and Widdicombe, J.G. 1995. Regulation of human airway surface liquid. Respir. Physiol. 99:3-12. 